\title{
Prolonged survival with adrenal cortical carcinoma
}

\author{
K. M. SHAW \\ M.R.C.P. \\ J. L. PETERS
F.R.C.S.
}

\author{
C. FISHER \\ M.R.C.Path. \\ E. J. Ross \\ F.R.C.P.
}

\section{University College Hospital, London}

\begin{abstract}
Summary
Recurrence of adrenal cortical carcinoma 22 years after first presentation is reported in a 54-year-old woman with Cushing's syndrome. This tumour is normally associated with poor prognosis and prolonged survival is rare. Although the patient had remained well with apparent 'cure' in the intervening years, it is suggested that hormonal changes related to the menopause and the development of primary hypoadrenalism stimulated recurrence of malignant activity and return of Cushingoid features.
\end{abstract}

\section{Introduction}

Carcinoma of the adrenal cortex is rare, affecting about 1/500 000 persons (Hutter and Kayhoe, 1966). Untreated, the disease is rapidly fatal with a mean survival of 3 months (Macfarlane, 1958). Although $25 \%$ of patients can be 'cured' of their Cushing's syndrome by surgical removal of the primary adrenal tumour (Liddle, 1974), treatment is generally unsatisfactory, with a high early recurrence rate after surgery and a poor response to medical therapy.

Long-term survival with relapse is unusual and only the very rare late recurrence up to 16 years after initial presentation has been recorded (Harrison and Abelson, 1952). The authors believe the following case report of Cushing's syndrome secondary to tumour recurrence after an interval of 22 years to be highly exceptional.

\section{Case report}

A clinical diagnosis of Cushing's syndrome was made in 1952 when the patient, a female then aged 32 years, presented with a one-year history of amenorrhoea, weight gain and hirsutism. The only laboratory test available at that time, urinary 17ketosteroids, gave a value of $183 \mu \mathrm{mol} / 24 \mathrm{hr}$ (normal 20-50) with an elevated B fraction (35\%; normal $<10 \%$ ). Intravenous pyelography showed the left kidney to be depressed and rotated by a mass on the superomedial aspect, and pneumatography confirmed

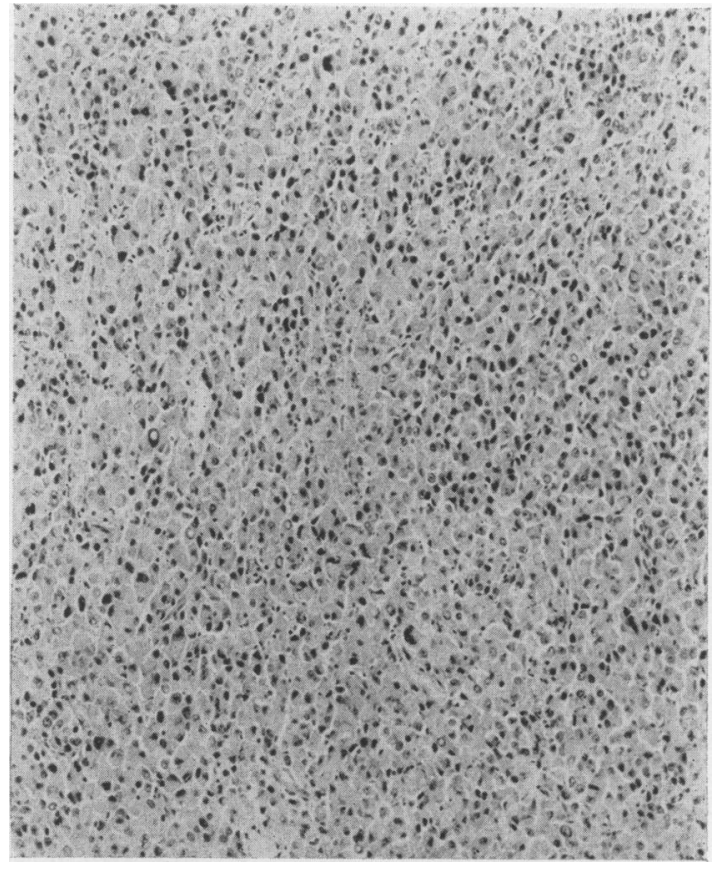

FIG. 1. Primary adrenal cortical carcinoma (1952) showing sheets of cells with abundant cytoplasm moderate nuclear pleomorphism and very scanty mitoses $(\times 140)$.

the presence of a large discrete round mass in the left paravertebral gutter below the diaphragm.

At laparotomy (W. R. Merrington) a vascular spherical tumour lying above the left kidney was excised. Large amounts of 17-ketosteroids were identified in the tumour perfusate, while the histology showed an epithelial tumour of the adrenal cortex of low grade malignancy (Fig. 1). At operation a small tear in the diaphragm was repaired and a small, transient left pleural effusion followed. After removal of the tumour there was a marked fall in 
urinary ketosteroids $(42 \mu \mathrm{mol} / 24 \mathrm{hr}$ with no B fraction) and the Cushingoid features regressed completely over the subsequent 2 months.

In 1972 at the age of 52 years, hysterectomy and oöphorectomy were performed for menorrhagia; otherwise the patient remained well for 22 years after removal of the adrenal carcinoma until 1974, when she noticed a return of her previous symptoms of weight gain, facial rounding and hirsutism. Plasma cortisol measurements showed loss of diurnal rhythm with an elevated midnight level $(290 \mathrm{nmol} / \mathrm{l})$ and complete failure to suppress with dexamethasone. Chest X-ray showed a mass in the left lower lobe of the lung. Thoracotomy was performed (J. C. R. Lincoln) and the left lower lobe was excised with the mass. Histology was identical to that of the primary tumour of 1952 (Fig. 2). The clinical features of Cushing's syndrome regressed and, although the plasma cortisol concentration returned to normal, a rise in plasma corticotrophin to $81 \mu \mathrm{g} / \mathrm{ml}$ was subsequently recorded.

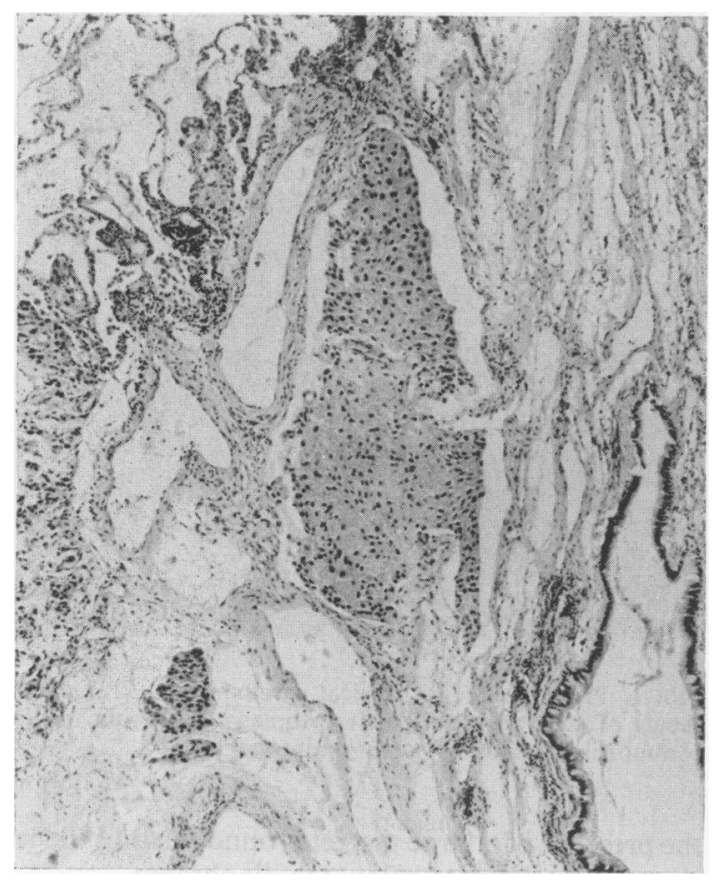

FIG. 2. Metastatic adrenal cortical carcinoma in pulmonary lymphatics (1974) ( $\times 70)$.

Two years later, in January 1976, symptoms returned and a further opacity in the left lung was noted. At completion pneumonectomy (J. C. R. Lincoln) another tumour of the same histology was removed from the left upper lobe, following which the plasma cortisol concentration again diminished (9.00 a.m. $18 \mathrm{nmol} / 1$; midnight $9 \mathrm{nmol} / \mathrm{l})$ associated with a rise in plasma corticotrophin $(80 \mu \mathrm{g} / \mathrm{ml})$.

To establish whether residual tumour remained $c$ at the original primary site in the abdomen, lapar- $\overrightarrow{\bar{F}}$ otomy, covered with hydrocortisone therapy, was 7 performed (G. L. Bunton). Necrotic lesions of $\frac{\mathrm{C}}{\sigma}$ adrenal cortical carcinoma tissue, indistinguishable $\overline{\bar{c}}$ histologically from that of 1952, were found scattered $\widehat{\nabla}$ in the mesentery and could not be resected. The left adrenal gland was noted to be absent and the os original adrenal bed was tumour-free. The right $\vec{\circ}$ adrenal gland was atrophic.

Following laparotomy treatment with hydrocortisone was gradually withdrawn and within 8 days of stopping therapy the patient felt tired and weak, developed aches in her joints and became dizzy on standing. Her blood pressure fell to $100 / 70 \mathrm{mmHg}$ lying and $90 / 0$ standing. The 9.00 a.m. plasma cortisol was $3 \mathrm{nmol} / \mathrm{l}$ with no rise after stimulation with tetracosactrin. Treatment with of hydrocortisone was therefore continued, and to this was added oestrogen therapy with ethinyloestradiol. On this drug regime the patient is well 4 years later (1979) without further evidence of metastases in lung or elsewhere.

\section{Discussion}

Presenting at any age, carcinoma of the adrenat cortex is diagnosed twice as commonly in females than in males, probably because virilization is more easily recognized (Crowley, 1976). In a review of 138 patients with adrenal cortical carcinoma, Hutter and Kayhoe (1966) found evidence of local spread of tumour in $65 \%$ patients on first presentation, of metastatic spread to lungs in $53 \%$, to liver in $44 \%$ and to distant lymph nodes in $11 \%$.

Untreated, the prognosis is poor. Hutter and Kayhoe treated patients with the adrenolytic agent, $o, p^{\prime}-\mathrm{DDD}$, and of these, only $52 \%$ females and $38 \%$ males were alive at 4 years, with a mean survival of 56 and 19 months respectively. Further experience with $o, p^{\prime}-\mathrm{DDD}$ has confirmed its limited value in treatment (Orth and Liddle, 1971).

The postoperative (1951) pleural effusion in this patient is of interest and suggests tumour cells may have been introduced at the time of operation into the left pleural cavity, where they remained quiescent for the next 2 decades. The reappearance of Cushingoid features and evidence of tumour recurrence became apparent within 2 years of the surgical menopause. It is therefore possible that stimulation and regrowth of the tumour within the chest were determined by hormonal changes associated with the menopause, and for this reason the patient was treated with oestrogen. 
Steroid secretion by the tumour secondaries could have resulted in complete atrophy of the remaining adrenal gland. This might have established a vicious cycle, once the metastases were removed, allowing plasma corticotrophin levels to rise and thus stimulating further tumour recurrence.

Subsequent treatment with replacement doses of hydrocortisone has suppressed pituitary release of corticotrophin, and the patient on combined hydrocortisone and oestrogen therapy has remained well. She has now survived for 27 years after the removal of the primary tumour, and 5 years after metastases in the lung first became apparent.

\section{References}

Crowley, W.F. (1976) Case records of Massachusetts General Hospital. New Journal of Medicine, 295, 774.

Harrison, R.J. \& Abelson, D. (1952) Carcinoma of the adrenal cortex with endocrine manifestations. British Medical Journal, 1, 303.

HutTer, A.M. \& KAYHOE, D.E. (1966) Adrenal cortical carcinoma. American Journal of Medicine, 41, 572.

LIDDLE, G.W. (1974) The adrenal cortex. In: Textbook of Endocrinology (Ed. by Williams, R. H.), p. 259. W. B. Saunders Co., Philadelphia.

Macfarlane, D.A. (1958) Cancer of the adrenal cortex. Annals of the Royal College of Surgeons of England, 23, 155.

ORTH, D.N. \& LiDDLE, G.W. (1971) Results of treatment in 108 patients with Cushing's syndrome. New England Journal of Medicine, 285, 243. 